\title{
PROPERTIES OF SOLUTION SET OF STOCHASTIC INCLUSIONS $^{1}$
}

\author{
MICHAE KISIELEWICZ \\ Institute of Mathematics \\ Higher College of Engineering \\ Podgórna 50, 65-246 Zielona Góra, POLAND
}

\begin{abstract}
The properties of the solution set of stochastic inclusions $x_{t}-x_{s} \in c l{ }_{L^{2}}\left(\int_{s}^{t} F_{\tau}\left(x_{\tau}\right) d \tau+\int_{s}^{t} G_{\tau}\left(x_{\tau}\right) d w_{\tau}+\int_{s}^{t} \int_{\mathbb{R}^{n}} H_{\tau, z}\left(x_{\tau}\right) \tilde{\nu}(d \tau, d z)\right)$ are investigated. They are equivalent to properties of fixed points sets of appropriately defined set-valued mappings.
\end{abstract}

Key words: Stochastic inclusions, existence solutions, solution set, weak compactness.

AMS (MOS) subject classifications: $\quad 93 \mathrm{E} 03,93 \mathrm{C} 30$.

\section{INTRODUCTION}

There is a large number of papers (see for example [1], [4] and [5]) dealing with the existence of optimal controls of stochastic dynamical systems described by integral stochastic equations. Such problems can be described (see [10]) by stochastic inclusions $(S I(F, G, H))$ of the form

$$
x_{t}-x_{s} \in c l_{L^{2}}\left(\int_{s}^{t} F_{\tau}\left(x_{\tau}\right) d \tau+\int_{s}^{t} G_{\tau}\left(x_{\tau}\right) d w_{\tau}+\int_{s}^{t} \int_{\mathbb{R}^{n}} H_{\tau, z}\left(x_{\tau}\right) \tilde{\nu}(d \tau, d z)\right),
$$

where the stochastic integrals are defined by Aumann's procedure (see [7], [9]).

The results of the paper are concerned with properties of the set of all solutions to $S I(F, G, H)$. To begin with, we recall the basic definitions dealing with set-valued stochastic integrals and stochastic inclusions presented in [10]. We assume, as given, a complete filtered probability space $\left(\Omega, \mathcal{F},\left(\mathscr{F}_{t}\right)_{t \geq 0}, P\right)$, where a family $\left(\mathcal{F}_{t}\right)_{t \geq 0}$, of $\sigma$-algebras $\mathcal{F}_{t} \subset \mathcal{F}$ is assumed to be increasing: $\mathscr{F}_{s} \subset \mathscr{F}_{t}$ if $s \leq t$. We set $\mathbb{R}_{+}=[0, \infty)$, and $\mathfrak{B}_{+}$will denote the Borel $\sigma$-algebra on

\footnotetext{
${ }^{1}$ Received: April, 1993. Revised: July, 1993.
} 
$\mathbb{R}_{+}$. We consider set-valued stochastic processes $\left(F_{t}\right)_{t \geq 0},\left(g_{t}\right)_{t \geq 0}$ and

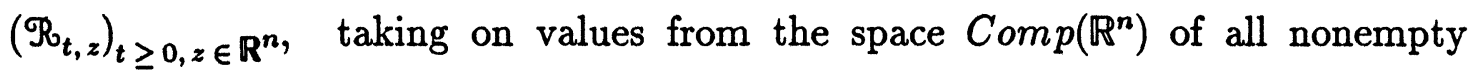
compact subsets of $n$-dimensional Euclidean space $\mathbb{R}^{n}$. They are assumed to be predictable and such that $E \int_{0}^{\infty}\left\|\mathcal{F}_{t}\right\|^{p} d t<\infty, \quad p \geq 1, \quad E \int_{0}^{\infty}\left\|\mathscr{G}_{t}\right\|^{2} d t<\infty$ and $E \int_{0}^{\infty} \int_{\mathbb{R}^{n}}\left\|\mathscr{R}_{t, z}\right\|^{2} d t q(d z)<\infty$, where $q$ is a measure on the Borel $\sigma$-algebra $\mathscr{B}^{n}$ of $\mathbb{R}^{n}$ and $\|A\|:=\sup \{|a|: a \in A\}, A \in \operatorname{Comp}\left(\mathbb{R}^{n}\right)$. The space $\operatorname{Comp}\left(\mathbb{R}^{n}\right)$ is considered with the Hausdorff metric $h$ defined in the usual way, i.e., $h(A, B)=\max \{\bar{h}(A, B), \bar{h}(B, A)\}, \quad$ for $\quad A, B \in \operatorname{Comp}\left(\mathbb{R}^{n}\right), \quad$ where $\quad \bar{h}(A, B)$ $=\{\operatorname{dist}(a, B): a \in A\}$ and $\bar{h}(B, A)=\{\operatorname{dist}(b, A): b \in B\}$. Although the classical theory of stochastic integrals (see [3], [8], [12]) usually deals with measurable and $\mathcal{F}_{t}$-adapted processes, it can be finally reduced (see [4], pp. 60-62) to predictable ones.

\section{BASIC DEFINITIONS AND NOTATIONS}

Throughout the paper we shall assume that a filtered complete probability space $\left(\Omega, \mathcal{F},\left(\mathcal{F}_{t}\right)_{t \geq 0}, P\right)$ satisfies the following usual hypotheses: $(i) \mathcal{F}_{0}$ contains all the $P$-null sets of $\mathcal{F}_{\text {, }}(i i) \mathcal{F}=\vee_{t \geq 0} \mathcal{F}_{t}$ and $(i i i) \quad \mathcal{F}_{t}=\bigcap_{u \rightarrow t} \mathcal{F}_{u}$, for all $t, 0 \leq t<\infty$. As usual, we consider a set $\mathbb{R}_{+} \times \Omega$ as a measurable space with the product $\sigma$-algebra $\mathfrak{B}_{+} \otimes \mathscr{F}$. Moreover, we introduce on $\mathbb{R}_{+} \times \Omega$ the predictable $\sigma$-algebra $\mathscr{P}$ generated by a semiring $\mathscr{T}$ of all predictable rectangles in $\mathbb{R}_{+} \times \Omega$ of the form $\{0\} \times A_{0}$ and $(s, t] \times A_{s}$, where $A_{0} \in \mathcal{F}_{0}$ and $\mathcal{A}_{s} \in \mathcal{F}_{s}$ for $s<t$ in $\mathbb{R}_{+}$. Similarly, besides the usual product $\sigma$-algebra on $\mathbb{R}_{+} \times \Omega \times \mathbb{R}^{n}$, we also introduce the predictable $\sigma$-algebra $\mathcal{P}^{n}$ generated by a semiring $9 \sigma^{n}$ of all sets of the form $\{0\} \times A_{0} \times D$ and $(s, t] \times A_{s} \times D$, with $A_{0} \in \mathcal{F}_{0}, A_{s} \in \mathcal{F}_{s}$ for $s<t$ in $\mathbb{R}_{+}$and $D \in \mathfrak{B}_{0}^{n}$, where $\mathfrak{B}_{0}^{n}$ consists of all Borel sets $D \subset \mathbb{R}^{n}$ such that their closure does not contain the point 0 .

An $n$-dimensional stochastic process $x$, understood as a function

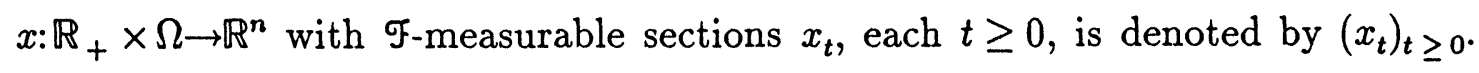
It is measurable (predictable) if $x$ is $\mathfrak{B}_{+} \otimes \mathscr{F}(\mathscr{P}$, resp.)-measurable. The process $\left(x_{t}\right)_{t \geq 0}$ is $\mathscr{F}_{t}$-adapted if $x_{t}$ is $\mathcal{F}_{t}$-measurable for $t \geq 0$. It is clear (see [3], [8], [11]) that every predictable process is measurable and $\mathcal{F}_{t^{-}}$-adapted. In what follows the Banach space $L^{p}\left(\mathbb{R}_{+} \times \Omega, \mathcal{P}, d t \times P, \mathbb{R}^{n}\right), p \geq 1$, with the norm $\|\cdot\|_{\ell_{n}^{p}}$ defined in the usual way, will be denoted by $L_{n}^{p}$. Similarly, the Banach spaces 
$L^{p}\left(\Omega, \mathscr{F}_{t}, P, \mathbb{R}^{n}\right)$ and $L^{p}\left(\Omega, \mathscr{F}, P, \mathbb{R}^{n}\right)$ with the usual norm $\|\cdot\|_{L_{n}^{p}}$ are denoted by $L_{n}^{p}\left(\mathscr{F}_{t}\right)$ and $L_{n}^{p}(\mathscr{F})$, respectively.

Throughout the paper, by $\left(w_{t}\right)_{t \geq 0}$, we mean a one-dimensional $F_{t^{-}}$ Brownian motion starting at 0 , i.e., such that $P\left(w_{0}=0\right)=1$. By $\nu(t, A)$ we denote a $\mathscr{F}_{t}$-Poisson measure on $\mathbb{R}_{+} \times \mathscr{B}^{n}$, and then define a $\mathscr{F}_{t}$-centered Poisson measure $\widetilde{\nu}(t, A), t \geq 0, A \in \mathfrak{B}^{n}$, by taking $\widetilde{\nu}(t, A)=\nu(t, A)-t q(A), t \geq 0, A \in \mathfrak{B}^{n}$, where $q$ is a measure on $\mathfrak{G}^{n}$ such that $E \nu(t, B)=t q(B)$ and $q(B)<\infty$ for $B \in \mathbb{T}_{0}^{n}$.

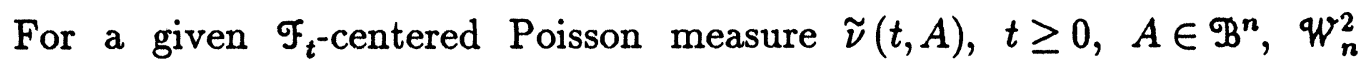
denotes the space $L^{2}\left(\mathbb{R}_{+} \times \Omega \times \mathbb{R}^{n}, \Phi^{n}, d t \times P \times q\right)$, with the norm $\|\cdot\|_{\mathscr{W}_{n}^{2}}$ defined in the usual way. We shall also consider the Banach spaces $L^{p}\left(\mathbb{R}_{+}, \mathscr{B}_{+}, d t, \mathbb{R}_{+}\right), p \geq 1$ and $L^{2}\left(\mathbb{R}_{+} \times \mathbb{R}^{n}, \mathscr{B}_{+} \otimes \mathfrak{B}^{n}, d t \times q, \mathbb{R}_{+}\right)$, with the usual norms by $|\cdot|_{p}$ and $\|\cdot\|_{2}$, respectively. They will be denoted by $L^{p}\left(\mathscr{B}_{+}\right)$and $L^{2}\left(\mathscr{B}_{+} \times \mathscr{B}^{n}\right)$, respectively. Finally, by $\mathcal{M}_{n}^{p}(\mathcal{P}), p \geq 1$ and $\mathcal{M}_{n}^{2}\left(\mathscr{P}^{n}, q\right)$ we shall denote the families of all $\mathcal{P}^{-}$-measurable and $\mathscr{\Phi}^{n}$-measurable functions $f: \mathbb{R}_{+} \times \Omega \rightarrow \mathbb{R}^{n}$ and $h: \mathbb{R}_{+} \times \Omega \times \mathbb{R}^{n} \rightarrow \mathbb{R}^{n}$, respectively, such that $\int_{0}^{\infty}\left|f_{t}\right|^{p} d t<\infty$ and $\int_{0 \mathbb{R}^{n}}^{\infty}\left|h_{t, z}\right|^{2} d t q(d z)<\infty$, a.s. Elements of $\mathcal{M}_{n}^{p}\left(\Im^{P}\right), p \geq 1$ and $\mathcal{M}_{n}^{2}\left(\mathcal{P}^{n}, q\right)$ will

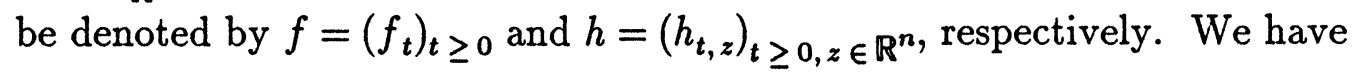

and

$$
\begin{gathered}
\mathcal{L}_{n}^{p}=\left\{f \in \mathcal{M}^{p}(\Phi): E \int_{0}^{\infty}\left|f_{t}\right|^{p} d t<\infty\right\}, p \geq 1, \\
W_{n}^{2}=\left\{h \in \mathcal{M}^{2}\left(\Phi^{n}, q\right): E \int_{0}^{\infty} \int_{\mathbb{R}^{n}}\left|h_{t, z}\right|^{2} d t q(d z)<\infty\right\} .
\end{gathered}
$$

Given $\quad g \in \mathcal{M}^{2}(\Phi) \quad$ and $\quad h \in \mathscr{M}^{2}\left(\Phi^{n}, q\right), \quad$ by $\quad\left(\int_{0}^{t} g_{\tau} d w_{\tau}\right)_{t \geq 0} \quad$ and $\left(\int_{0}^{t} \int_{\mathbb{R}^{n}} h_{\tau, z^{2}} \tilde{\nu}(d \tau, d z)\right)_{t \geq 0}$, we denote their stochastic integrals with respect to a $\digamma_{t^{-}}$ Brownian motion $\left(w_{t}\right)_{t \geq 0}$ and a $\sigma_{t}$-centered Poisson measure $\tilde{\nu}(t, A), t \geq 0$, $A \in \mathfrak{B}^{n}$, respectively. These integrals, understood as $n$-dimensional stochastic processes, have quite similar properties (see [6]).

Let us denote by $D$ the family of all $n$-dimensional $\digamma_{t}$-adapted cádlág processes $\left(x_{t}\right)_{t \geq 0}$ such that

$$
\operatorname{Esup}_{t \geq 0}\left|x_{t}\right|^{2}<\infty
$$

and

$$
\lim _{\delta \rightarrow 0} \sup _{t \geq 0} \sup _{t \leq s \leq t+\delta} E\left|x_{t}-x_{s}\right|^{2}=0 .
$$

Recall that an $n$-dimensional stochastic process is said to be a cádlág process if it has almost all sample paths right continuous with finite left limits. The space $D$ is considered as a normed space with the norm $\|\cdot\|_{\ell}$ defined by 
$\|\xi\|_{\ell}=\left\|\sup _{t \geq 0}\left|\xi_{t}\right|\right\|_{L_{1}^{2}}$ for $\xi=\left(\xi_{t}\right)_{t \geq 0} \in D . \quad$ It can be verified that $\left(D,\|\cdot\|_{\ell}\right)$ is a Banach space.

Given $0 \leq \alpha<\beta<\infty$ and $\left(x_{t}\right)_{t \geq 0} \in D$ let $x^{\alpha, \beta}=\left(x_{t}^{\alpha, \beta}\right)_{t \geq 0}$ be defined by $x_{t}^{\alpha, \beta}=x_{\alpha}$ and $x_{t}^{\alpha, \beta}=x_{\beta}$ for $0 \leq t \leq \alpha$ and $t \geq \beta$, respectively, and $x_{t}^{\alpha, \beta}=x_{t}$ for $\alpha \leq t \leq \beta$. It is clear that $D^{\alpha, \beta}:=\left\{x^{\alpha, \beta}: x \in D\right\}$ is a linear subspace of $D$, closed in the $\|\cdot\|_{\ell}$-norm topology. Then $\left(D^{\alpha, \beta},\|\cdot\|_{\ell}\right)$ is also a Banach space. Finally, as usual, by $\sigma\left(D, D^{*}\right)$ we shall denote a weak topology on $D$.

In what follows we shall deal with upper and lower semicontinuous set-

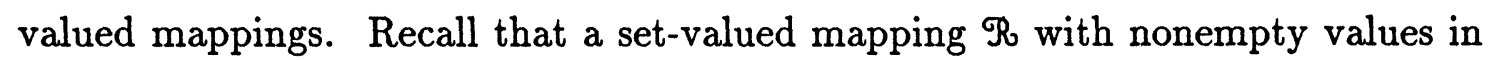
a topological space $\left(Y, \mathscr{T}_{Y}\right)$ is said to be upper (lower) semicontinuous [u.s.c. (1.s.c.)] on a topological space $\left(X, \mathcal{T}_{X}\right)$ if $\Re^{-}(C):=\{x \in X: \mathscr{R}(x) \cap C \neq \emptyset\}$ $\left(\Re_{-}(C):=\left\{x \in X: \Re_{(}(x) \subset C\right\}\right)$ is a closed subset of $X$ for every closed set $C \subset Y$. In particular, for $\Re$ defined on a metric space $(\mathscr{G}, d)$ with values in $\operatorname{Comp}\left(\mathbb{R}^{n}\right), \quad$ it is equivalent (see [9]) to $\lim _{n \rightarrow \infty} \bar{h}\left(\mathscr{R}\left(x_{n}\right), \mathscr{R}(x)\right)=0$ $\left(\lim _{n \rightarrow \infty} \bar{h}\left(\mathscr{R}(x), \Re_{R}\left(x_{n}\right)\right)=0\right)$ for every $x \in \mathscr{S}$ and every sequence $\left(x_{n}\right)$ of 96 converging to $x$. If, moreover, $\Re$ takes convex values then it is equivalent to upper (lower) semicontinuity of a real-valued function $s(p, \mathscr{B}(\cdot))$ on $\mathbb{R}^{n}$ for every $p \in \mathbb{R}^{n}$, where $s(\cdot, A)$ denotes a support function of a set $A \in \operatorname{Comp}\left(\mathbb{R}^{n}\right)$. In what follows, we shall need the follow well-known (see [9]) fixed point and continuous selection theorems.

Theorem (Schauder, Tikhonov): $\quad$ Let $\left(X, \mathcal{T}_{X}\right)$ be a locally convex topological Hausdorff space, 96 a nonempty compact convex subset of $X$ and $f$ a continuous mapping of 96 into itself. Then $f$ has a fixed point in 96.

Theorem (Covitz, Nadler): Let $(\mathscr{G}, d)$ be a complete metric space and $\mathfrak{R}_{0} \mathscr{\mathscr { C }} \rightarrow \mathrm{Cl}(\mathscr{G})$ a set-valued contraction mapping, i.e., such that $H(\Re(x), \mathscr{R}(y)) \leq \lambda d(x, y)$ for $x, y \in \mathscr{G}$ with $\lambda \in[0,1)$, where $H$ is the Hausdorff metric induced by the metric $d$ on the space $C l(96)$ of all nonempty closed bounded subsets of 96 . Then there exists $x \in 9$ such that $x \in \mathscr{B}(x)$.

Theorem (Kakutani, Fan):

Let $\left(X, \mathcal{T}_{X}\right)$ be a locally convex topological Hausdorff space, $\mathscr{T}$ a nonempty compact convex subset of $X$ and $\mathrm{CCl}(96)$ a family of all nonempty closed convex subsets of 96 . If $\Re: 96 \rightarrow C C l(96)$ is u.s.c. on $\mathscr{T}$ then there exists $x \in \mathscr{G}$ such that $x \in \mathscr{R}(x)$. 
Theorem (Michael): Let $\left(X, \mathcal{T}_{X}\right)$ be a paracompact space and let $\mathscr{R}$ be a set-valued mapping from $X$ to a Banach space $(Y,\|\cdot\|)$ whose values are closed and convex. Suppose, further $\mathscr{B}$ is l.s.c. on $X$. Then there is a continuous function $f: X \rightarrow Y$ such that $f(x) \in \mathscr{R}(x)$, for each $x \in X$.

\section{SET-VALUED STOCHASTIC INTEGRALS}

Let $g=\left(g_{t}\right)_{t \geq 0}$ be a set-valued stochastic process with values in

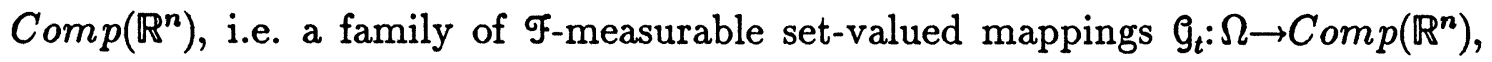
$t \geq 0$. We call $g$ measurable (predictable) if it is $\mathfrak{B}_{+} \otimes \mathscr{F}(\mathscr{P}$, resp.)-measurable. Similarly, $g$ is said to be $\mathcal{F}_{t}$-adapted if $g_{t}$ is $\mathcal{F}_{t}$-measurable for each $t \geq 0$. It is clear that every predictable set-valued stochastic process is measurable and $\boldsymbol{F}_{t^{-}}$ adapted. It follows from the Kuratowski and Ryll-Nardzewski measurable selection theorem (see [9]) that every measurable (predictable) set-valued process with nonempty compact values possesses a measurable (predictable) selector. We shall also consider $\mathfrak{B}_{+} \otimes \mathscr{F} \otimes \mathscr{B}^{n}$ and $\mathscr{P}^{n}$-measurable set-valued mappings $\mathscr{R}: \mathbb{R}_{+} \times \Omega \times \mathbb{R}^{n} \rightarrow C l\left(\mathbb{R}^{n}\right)$. They will be denoted as families $\left(\mathscr{R}_{t, z}\right)_{t \geq 0, z} \in \mathbb{R}^{n}$ and called measurable and predictable, respectively set-valued stochastic processes depending on a parameter $z \in \mathbb{R}^{n}$. The process $\mathscr{B}=\left(\mathscr{R}_{t, z}\right)_{t \geq 0, z \in \mathbb{R}^{n}}$ is said to be $\mathscr{F}_{t}$-adapted if $\mathscr{R}_{t, z}$ is $\mathscr{F}_{t}$-measurable for each $t \geq 0$ and $z \in \mathbb{R}^{n}$.

Denote by $\mathcal{M}_{s-v}^{p}(\Phi), p \geq 1$, and $\mathcal{M}_{s-v}^{2}\left(\Phi^{n}, q\right)$ the families of all set-valued

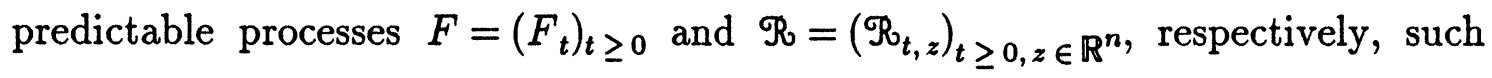
that $E \int_{0}^{\infty}\left\|F_{t}\right\|^{p} d t<\infty$ and $E \int_{0 \mathbb{R}^{n}}^{\infty}\left\|\mathscr{R}_{t, z}\right\|^{2} d t q(z)<\infty$. Immediately from the Kuratowski and Ryll-Nardzewski measurable selection theorem it follows that for every $F \in \mathcal{M}_{s-v}^{p}\left({ }^{P}\right), p \geq 1$, and $\Re \in \mathcal{H}_{s-v}^{2}\left(\mathscr{(}^{n}, q\right)$ the sets

$$
\varphi^{p}(F):=\left\{f \in \mathcal{L}_{n}^{p}: f_{t}(\omega) \in F_{t}(\omega), d t \times P \text { - a.e. }\right\}
$$

and

$$
\varphi_{q}^{2}\left(\mathscr{R}_{\mathrm{R}}\right):=\left\{h \in \mathcal{W}_{n}^{2}: h_{t, z}(\omega) \in \mathscr{R}_{t, z}(\omega), d t \times P \times q-\text { a.e. }\right\}
$$

are nonempty.

Given set-valued processes $F=\left(F_{t}\right)_{t \geq 0} \in \mathcal{H}_{s-v}^{p}(\mathcal{P}), \quad \mathcal{g}=\left(\mathcal{G}_{t}\right)_{t \geq 0}$

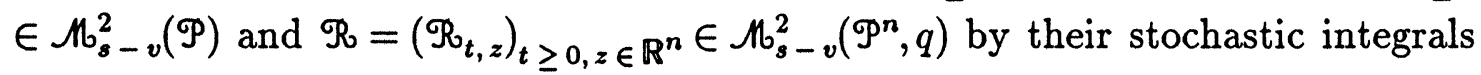
$g F, \quad g g$ and $\mathcal{T} \mathscr{R}$ we mean families $g F=\left(g_{t} F\right)_{t \geq 0}, \quad g g=\left(g_{t} g\right)_{t \geq 0}$, and $\mathcal{T}_{\mathbb{R}}=\left(\mathcal{T}_{t} \mathscr{R}_{t}\right)_{t \geq 0}$ subsets of $L_{n}^{p}\left(\mathcal{F}_{t}\right), p \geq 1$ and $L_{n}^{2}\left(\mathcal{F}_{t}\right)$, respectively, defined by 
$g_{t} F=\left\{g_{t} f: f \in \varphi^{p}(F)\right\}, \quad g_{t} g=\left\{g_{t} g: g \in \varphi^{2}(g)\right\}$ and $\mathcal{T}_{t} \mathscr{R}=\left\{\mathcal{G}_{t} h: h \in \mathscr{\varphi}_{q}^{2}\left(\mathscr{F}_{0}\right)\right\}$, where $g_{t} f=\int_{0}^{t} f_{s} d s, g_{t} g=\int_{0}^{t} g_{s} d w_{s}$ and $\mathscr{T}_{t} h=\int_{0}^{t} \int_{\mathbb{R}^{n}} h_{s, z} \tilde{\nu}(d s, d z)$. Given $0 \leq \alpha<\beta<\infty$, we also define $\int_{\alpha}^{\beta} F_{s} d s=\left\{\int_{\alpha}^{\beta} f_{s} d s: f \in \varphi^{p}(F)\right\}, \int_{\alpha}^{\beta} g_{s} d w_{s}=\left\{\int_{\alpha}^{\beta} g_{s} d w_{s}: g \in \varphi^{2}(g)\right\}$ and $\int_{\alpha}^{\beta} \int_{\mathbb{R}^{n}} \mathscr{R}_{s, z} \tilde{\nu}(d s, d z)=\left\{\int_{\alpha}^{\beta} \int_{\mathbb{R}^{n}} h_{s, z} \tilde{\nu}(d s, d z): h \in \mathscr{\varphi}^{2}\left(\mathscr{R}_{\mathcal{Q}}\right)\right\}$. The following properties of set-valued stochastic integrals are given in [10].

Proposition 1: Let $F \in \mathcal{M}_{s-v}^{p}(\mathcal{P}), \quad p \geq 1, \quad g \in \mathcal{M}_{s-v}^{2}(\mathcal{P})$ and $\mathscr{B} \in \mathcal{M}_{s-v}^{2}\left(\Phi^{n}, q\right)$. Then

(i) $g_{t} \mathcal{G}$ and $\mathcal{T}_{t} \mathfrak{R}$ are closed subsets of $L_{n}^{2}\left(\mathcal{F}_{t}\right)$ for each $t \geq 0$.

(ii) If, moreover, $F, \mathcal{G}$ and $\mathscr{B}$ take on convex values then $g_{t} F, g_{t} g$ and $\mathcal{T}_{t} \Re$ are convex and weakly compact in $L_{n}^{p}\left(\mathscr{F}_{t}\right)$ and $L_{n}^{2}\left(\mathscr{F}_{t}\right)$, respectively, for each $t \geq 0$.

Proposition 2: Let $\quad F \in \mathcal{M}_{s-v}^{2}(\mathscr{P}), \quad g \in \mathcal{M}_{s-v}^{2}(\mathscr{P}) \quad$ and $\mathscr{B} \in \mathcal{M}_{s-v}^{2}\left(\Phi^{n}, q\right)$. Assume $\left(x_{t}\right)_{t \geq 0} \in D$ is such that

$$
x_{t}-x_{s} \in c L_{L^{2}}\left(\int_{s}^{t} F_{\tau} d \tau+\int_{s}^{t} \mathfrak{g}_{\tau} d w_{\tau}+\int_{s}^{t} \int_{\mathbb{R}^{n}} \mathscr{R}_{\tau, z} \tilde{\nu}(d \tau, d z)\right)
$$

for every $0 \leq s<t<\infty$. Then for every $\epsilon>0$ there are $f^{\epsilon} \in \varphi^{p}(F), g^{\epsilon} \in \varphi^{2}(g)$ and $h^{\epsilon} \in \varphi_{q}^{2}(\mathscr{R})$ such that

$$
\sup _{t \geq 0}\left\|\left|\left(x_{t}-x_{0}\right)-\left(\int_{0}^{t} f_{\tau}^{\epsilon} d \tau+\int_{0}^{t} g_{\tau}^{\epsilon} d w_{\tau}+\int_{0}^{t} \int_{\mathbb{R}^{n}} h_{\tau, z}^{\epsilon} \tilde{\nu}(d \tau, d z)\right)\right|\right\|_{L^{2}} \leq \epsilon .
$$

Proposition 3: Assume $F \in \mathcal{M}_{s-v}^{2}(\Phi), \quad g \in \mathcal{M}_{s-v}^{2}(\Phi)$ and $\mathscr{R}_{B} \in \mathcal{M}_{s-v}^{2}\left(\mathscr{P}^{n}, q\right)$ take on convex values and let $\left(x_{t}\right)_{t \geq 0} \in D$. Then

$$
x_{t}-x_{s} \in \int_{s}^{t} F_{\tau} d \tau+\int_{s}^{t} g_{\tau} d w_{\tau}+\int_{s}^{t} \int_{\mathbb{R}^{n}} \mathscr{R}_{\tau, z} \tilde{\nu}(d \tau, d z)
$$

for $0 \leq s<t<\infty$ if and only if there are $f \in \varphi^{2}(F), g \in \varphi^{2}(\mathcal{G})$ and $h \in \varphi_{q}^{2}\left(\Re_{R}\right)$ such that

$$
x_{t}=x_{0}+\int_{0}^{t} f_{\tau} d \tau+\int_{0}^{t} g_{\tau} d w_{\tau}+\int_{0}^{t} \int_{\mathbb{R}^{n}} h_{\tau, z} \tilde{\nu}(d \tau, d z), \text { a.s. for each } t \geq 0 .
$$




\section{STOCHASTIC INCLUSIONS}

Let $\quad F=\left\{\left(F_{t}(x)\right)_{t \geq 0}: x \in \mathbb{R}^{n}\right\}, \quad G=\left\{\left(G_{t}(x)\right)_{t \geq 0}: x \in \mathbb{R}^{n}\right\} \quad$ and $\quad H=$

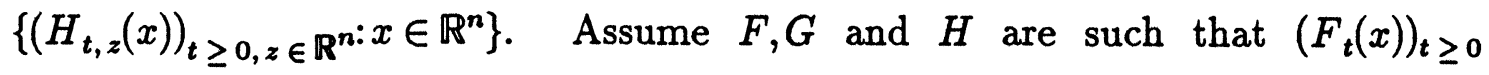

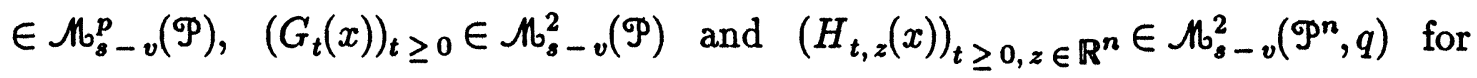
each $x \in \mathbb{R}^{n}$.

By a stochastic inclusion, denoted by $S I(F, G, H)$, corresponding to $F, G$ and $H$ given above, we mean the relation

$$
x_{t}-x_{s} \in \operatorname{cl}_{L^{2}}\left(\int_{s}^{t} F_{\tau}\left(x_{\tau}\right) d \tau+\int_{s}^{t} G_{\tau}\left(x_{\tau}\right) d w_{\tau}+\int_{s}^{t} \int_{\mathbb{R}^{n}} H_{\tau, z}\left(x_{\tau}\right) \widetilde{\nu}(d \tau, d z)\right)
$$

that is to be satisfied for every $0 \leq s<t<\infty$ by a stochastic process $x=\left(x_{t}\right)_{t \geq 0} \in D \quad$ such that $F \circ m x \in \mathcal{M}_{s-v}^{p}(\mathcal{P}), \quad G \circ m x \in \mathcal{H}_{s-v}^{2}(\mathcal{P})$ and $H \circ m x \in \mathcal{M}_{s-v}^{2}\left(\Phi^{n}, q\right)$, where $F \circ m x=\left(F_{t}\left(x_{t}\right)\right)_{t \geq 0}, G \circ m x=\left(G_{t}\left(x_{t}\right)\right)_{t \geq 0}$ and

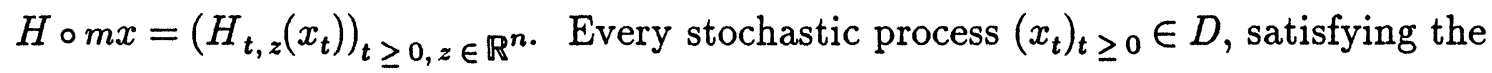
conditions mentioned above, is said to be global solution to $S I(F, G, H)$.

Corollary 1: If $F, G$ and $H$ take on convex values then $S I(F, G, H)$ has a form

$$
x_{t}-x_{s} \in \int_{s}^{t} F_{\tau}\left(x_{\tau}\right) d \tau+\int_{s}^{t} G_{\tau}\left(x_{\tau}\right) d w_{\tau}+\int_{s}^{t} \int_{\mathbb{R}^{n}} H_{\tau, z}\left(x_{\tau}\right) \tilde{\nu}(d \tau, d z)
$$

and $\left(x_{t}\right)_{t \geq 0} \in D$ is a global solution to $S I(F, G, H)$ if and only if there are $f \in \varphi^{2}(F \circ m x), g \in \varphi^{2}(G \circ m x)$ and $h \in \varphi_{q}^{2}(H \circ m x)$ such that

$$
x_{t}=x_{0}+\int_{0}^{t} f_{\tau} d \tau+\int_{0}^{t} g_{\tau} d w_{\tau}+\int_{0}^{t} \int_{\mathbb{R}^{n}} h_{\tau, z} \widetilde{\nu}(d \tau, d z), \text { a.s. for each } t \geq 0 \text {. }
$$

Given $0 \leq \alpha<\beta<\infty$, a stochastic process $\left(x_{t}\right)_{t \geq 0} \in D$ is said to be a local solution to $S I(F, G, H)$ on $[\alpha, \beta]$ if

$$
x_{t}-x_{s} \in c l_{L^{2}}\left(\int_{s}^{t} F_{\tau}\left(x_{\tau}\right) d \tau+\int_{s}^{t} G_{\tau}\left(x_{\tau}\right) d w_{\tau}+\int_{s}^{t} \int_{\mathbb{R}^{n}} H_{\tau, z}\left(x_{\tau}\right) \tilde{\nu}(d \tau, d z)\right)
$$

for $\alpha \leq s<t \leq \beta$.

Corollary 2: A stochastic process $\left(x_{t}\right)_{t \geq 0} \in D$ is a local solution to $S I(F, G, H)$ on $[\alpha, \beta]$ if and only if $x^{\alpha, \beta}$ is a global solution to $S I\left(F^{\alpha \beta}, G^{\alpha \beta}, H^{\alpha \beta}\right)$, where $F^{\alpha \beta}=\mathbf{I}_{[\alpha, \beta]} F, G^{\alpha \beta}=\mathbf{I}_{[\alpha, \beta]} G$ and $H^{\alpha \beta}=\mathbf{I}_{[\alpha, \beta]} H$. 
A stochastic process $\left(x_{t}\right)_{t \geq 0} \in D$ is called a global (local on $[\alpha, \beta]$, resp.) solution to an initial value problem for stochastic inclusion $S I(F, G, H)$ with an initial condition $y \in L^{2}\left(\Omega, \mathcal{F}_{0}, \mathbb{R}^{n}\right)\left(y \in L^{2}\left(\Omega, \mathcal{F}_{\alpha}, \mathbb{R}^{n}\right)\right.$, resp. $)$ if $\left(x_{t}\right)_{t \geq 0}$ is a global (local on $[\alpha, \beta]$, resp.) solution to $S I(F, G, H)$ and $x_{0}=y\left(x_{\alpha}=y\right.$, resp.). An initial-value problem for $S I(F, G, H)$ mentioned above will be denoted by $S I_{y}(F, G, H)\left(S I_{y}^{\alpha, \beta}(F, G, H)\right.$, resp.). In what follows, we denote a set of all global (local on $[\alpha, \beta]$, resp.) solutions to $S I_{y}(F, G, H)$ by $\Lambda_{y}(F, G, H)$ $\left(\Lambda_{y}^{\alpha, \beta}(F, G, H)\right.$, resp.).

Suppose $F, G$ and $H$ satisfy the following conditions $\left(\mathcal{A}_{1}\right)$ :

(i) $F=\left\{\left(F_{t}(x)\right)_{t \geq 0}: x \in \mathbb{R}^{n}\right\}, G=\left\{\left(G_{t}(x)\right)_{t \geq 0}: x \in \mathbb{R}^{n}\right\}$ and $H=$

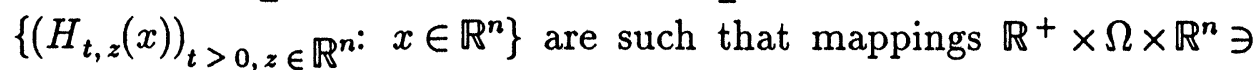
$(t, \omega, x) \rightarrow F_{t}(x)(\omega) \in C l\left(\mathbb{R}^{n}\right), \quad \mathbb{R}_{t} \times \Omega \times \mathbb{R}^{n} \ni(t, \omega, x) \rightarrow G_{t}(x)(\omega) \in C l\left(\mathbb{R}^{n}\right)$ and $\mathbb{R}_{t} \times \Omega \times \mathbb{R}^{n} \times \mathbb{R}^{n} \ni(t, \omega, z, x) \rightarrow H_{t, z}(x)(\omega) \in C l\left(\mathbb{R}^{n}\right)$ are $\mathscr{P} \otimes \mathscr{B}^{n}$ and $\mathscr{P}^{n} \otimes \mathfrak{B}^{n}$-measurable, respectively.

(ii) $\quad\left(F_{t}(x)\right)_{t \geq 0},\left(G_{t}(x)\right)_{t \geq 0}, \quad\left(H_{x, z}(x)\right)_{t \geq 0, z \in \mathbb{R}^{n}}$ are uniformly $p$ - and square-integrable bounded, respectively, i.e.,

$$
\begin{gathered}
\left(\sup _{x \in \mathbb{R}^{n}}\left\|F_{t}(x)\right\|\right)_{t \geq 0} \in \mathcal{L}_{1}^{p},\left(\sup _{x \in \mathbb{R}^{n}}\left\|G_{t}(x)\right\|\right)_{t \geq 0} \in \mathcal{L}_{1}^{2} \quad \text { and } \\
\left(\sup _{x \in \mathbb{R}^{n}}\left\|H_{t, z}(x)\right\|\right)_{t \geq 0, z \in \mathbb{R}^{n} \in W_{1}^{2} .}
\end{gathered}
$$

Corollary 3: For every $\left(x_{t}\right)_{t \geq 0} \in D$ and $F, G, H$ satisfying $\left(\mathcal{A}_{1}\right)$ one has $F \circ m x \in \mathcal{M}_{x-v}^{p}\left({ }^{(}\right), G \circ m x \in \mathcal{M}_{s-v}^{2}(\Phi)$ and $H \circ m x \in \mathcal{M}_{s-v}^{2}\left(\Phi^{n}, q\right)$.

Now define a linear continuous mapping $\Phi$ on $\mathcal{L}_{n}^{p} \times \mathcal{L}_{n}^{2} \times W_{n}^{2}$ by taking $\Phi(f, g, h)=\left(g_{t} f+g_{t} g+\mathcal{T}_{t} h\right)_{t \geq 0}$ to each $(f, g, h) \in \mathcal{L}_{n}^{p} \times \mathcal{L}_{n}^{2} \times \mathcal{W}_{n}^{2}$. It is clear that $\Phi$ maps $\mathcal{L}_{n}^{p} \times \mathcal{L}_{n}^{2} \times \mathcal{W}_{n}^{2}$ into $D$. Given above $F, G$ and $H$ satisfying $\left(\mathcal{A}_{1}\right)$, define a set-valued mapping 16 on $D$ by setting

$$
\mathscr{H}(x)=c l_{\ell}\left(\Phi\left(\varphi^{p}(F \circ m x) \times \varphi^{2}(G \circ m x) \times \varphi_{q}^{2}(H \circ m x)\right)\right)
$$

for $x=\left(x_{t}\right)_{t \geq 0} \in D$, where the closure is taken in the norm topology in $\left(D,\|\cdot\|_{\ell}\right)$. Similarly, for given $0 \leq \alpha<\beta<\infty$, we define a set-valued mapping $\jmath^{\alpha, \beta}$ on $D$ by taking

$$
{ }^{\prime} \mathcal{G}^{\alpha, \beta}(x)=c_{l}\left(\Phi\left(\varphi^{p}\left(F^{\alpha \beta} \circ m x\right) \times \varphi^{2}\left(G^{\alpha \beta} \circ m x\right) \times \varphi_{q}^{2}\left(H^{\alpha \beta} \circ m x\right)\right)\right.
$$

where $F^{\alpha \beta}, G^{\alpha \beta}$ and $H^{\alpha \beta}$ are as above.

Corollary 4: For every $F, G$ and $H$ taking on convex values and 
satisfying $\left(\mathcal{A}_{1}\right)$, one has $\mathcal{J}_{\mathcal{G}}(x)=\Phi\left(\varphi^{p}(F \circ m x) \times \varphi^{2}(G \circ m x) \times \varphi_{q}^{2}(H \circ m x)\right)$ and $\jmath_{6}^{\alpha, \beta}(y)=\Phi\left(\varphi^{p}\left(F^{\alpha \beta} \circ m x\right) \times \varphi^{2}\left(G^{\alpha \beta} \circ m x\right) \times \varphi_{q}^{2}\left(H^{\alpha \beta} \circ m x\right)\right)$ for $x \in D$.

Let $S(F, G, H)$ and $S^{\alpha, \beta}(F, G, H)$ denote the set of all fixed points of $J_{6}$ and $J_{G^{\alpha, \beta}}$, respectively. It will be shown below that $S^{\alpha, \beta}(F, G, H) \subset D^{\alpha, \beta}$. Immediately from Proposition 2 (see [10]) the following result follows.

Proposition 4: $\quad$ Assume $F, G$ and $H$ satisfy $\left(\mathcal{A}_{1}\right)$ and take on convex values. Then $\Lambda_{0}(F, G, H)=S(F, G, H)$ and $\Lambda_{0}^{\alpha, \beta}(F, G, H)=S^{\alpha, \beta}(F, G, H)$ for every $0 \leq \alpha<\beta<\infty$, respectively.

Proposition 5: Assume $F, G$ and $H$ satisfy $\left(\mathcal{A}_{1}\right)$ and let $0 \leq \alpha<\beta<\infty$. Then $x \in S^{\alpha, b}(F, G, H)$ if and only if

(i) $x_{t}=0$ a.s. for $t \in[0, \alpha]$,

(ii) $x_{t}=x_{\beta}$ a.s. for $t \geq \beta$,

(iii) for every $\epsilon>0$ there is $\left.\left(f^{\epsilon}, g^{\epsilon}, h^{\epsilon}\right) \in \varphi^{p}\left(F^{\alpha \beta} \circ m x\right) \times \varphi^{2}\left(G^{\alpha \beta} \circ m x\right) \times \varphi_{q}^{2}\left(H^{\alpha \beta} \circ m x\right)\right) \quad$ such that $\left\|\sup _{\alpha \leq t \leq \beta}\left|x_{t}-\Phi_{t}\left(f^{\epsilon}, g^{\epsilon}, h^{\epsilon}\right)\right|\right\|_{L_{1}^{2}}<\epsilon$.

Proof: $\quad(\Rightarrow)$ Let $x \in S^{\alpha, \beta}(F, G, H)$. By the definition of $\mathcal{J}_{6}^{\alpha, \beta}$, for every $\epsilon>0$, there is $\left.\left(f^{\epsilon}, g^{\epsilon}, h^{\epsilon}\right) \in \varphi^{p}\left(F^{\alpha \beta} \circ m x\right) \times \varphi^{2}\left(G^{\alpha \beta} \circ m x\right) \times \varphi_{q}^{2}\left(H^{\alpha \beta} \circ m x\right)\right)$ such that $\left\|x-\Phi\left(f^{\epsilon}, g^{\epsilon}, h^{\epsilon}\right)\right\|_{\ell}<\epsilon$. We have of course $\Phi_{t}\left(f^{\epsilon}, g^{\epsilon}, h^{\epsilon}\right)=0 \quad$ and $\Phi_{t}\left(f^{\epsilon}, g^{\epsilon}, h^{\epsilon}\right)=\Phi_{\beta}\left(f^{\epsilon}, g^{\epsilon}, h^{\epsilon}\right)$, a.s. for $0 \leq t \leq \alpha$ and $t \geq \beta$, respectively. Then

$$
\begin{gathered}
{\| \sup _{0 \leq t \leq \alpha}}\left|x_{t}\right|\left\|_{L_{1}^{2}}=\right\| \sup _{0 \leq t \leq \alpha}\left|x_{t}-\Phi_{t}\left(f^{\epsilon}, g^{\epsilon}, h^{\epsilon}\right)\right| \|_{L_{1}^{2}} \\
\leq\left\|x-\Phi\left(f^{\epsilon}, g^{\epsilon}, h^{\epsilon}\right)\right\|_{\ell}<\epsilon .
\end{gathered}
$$

and

$$
\begin{gathered}
\left\|\sup _{t \geq \beta}\left|x_{t}-x_{\beta}\right|\right\|_{L_{1}^{2}}=\left\|\sup _{t \geq \beta}\left|x_{t}-\Phi_{t}\left(f^{\epsilon}, g^{\epsilon}, h^{\epsilon}\right)\right|\right\|_{L_{1}^{2}} \\
+\left\|\sup _{t \geq \beta}\left|x_{\beta}-\Phi_{\beta}\left(f^{\epsilon}, g^{\epsilon}, h^{\epsilon}\right)\right|\right\|_{L_{1}^{2}}<2 \epsilon .
\end{gathered}
$$

Therefore, $\sup _{0} \leq t \leq \alpha\left|x_{t}\right|=0$ and $\sup _{t \geq \beta}\left|x_{t}-x_{\beta}\right|=0$ a.s.

By the properties of $\Phi\left(f^{\epsilon}, g^{\epsilon}, h^{\epsilon}\right),(i)$ and (ii), (iii) easily follow.

$(\Leftarrow)$ Conditions $(i)-($ iii $)$ imply

$$
\left\|x-\Phi\left(f^{\epsilon}, g^{\epsilon}, h^{\epsilon}\right)\right\|_{\ell}=\left\|\sup _{\alpha \leq t \leq \beta}\left|x_{t}-\Phi_{t}\left(f^{\epsilon}, g^{\epsilon}, h^{\epsilon}\right)\right|\right\|_{L_{1}^{2}}<\epsilon
$$


Therefore, $x \in c_{l} l^{\Phi\left(\varphi^{p}\left(F^{\alpha \beta} \circ m x\right) \times \varphi^{2}\left(G^{\alpha \beta} \circ m x\right) \times \varphi_{q}^{2}\left(H^{\alpha \beta} \circ m x\right)\right)}$.

Proposition 6: $\quad$ Assume $F, G$ and $H$ satisfy $\left(\mathcal{A}_{1}\right)$ and let $\left(\tau_{n}\right)_{n=1}^{\infty}$ be a sequence of positive numbers increasing to $+\infty$. If $x^{1} \in S^{0, \tau_{1}}(F, G, H)$ and $x^{n+1} \in x_{\tau_{n}}^{n}+S^{\tau_{n}, \tau_{n+1}}(F, G, H)$ for $n=1,2, \ldots$, then $x=\sum_{n=1}^{\infty} l_{\left(\tau_{n-1}, \tau_{n}\right)}\left(x^{n}\right.$ $-x_{\tau_{n-1}^{n-1}}^{n-1}$ ) belongs to $S(F, G, H)$, where $x_{0}^{0}=0$.

Proof: For every $n=1,2, \ldots$ one has $x^{n}-x_{\tau_{n-1}^{n-1}} \in S^{\tau_{n-1}, \tau_{n}}(F, G, H)$. Then, by Proposition 5, for every $n=1,2, \ldots$ and $\epsilon>0$ there is $\left(f^{n}, g^{n}, h^{n}\right) \in \varphi^{p}\left(F^{\tau_{n-1} \tau_{n}} \circ m x^{n}\right) \times \quad \varphi^{2}\left(G^{\tau_{n-1} \tau^{\tau}} \circ m x^{n}\right) \times \varphi_{q}^{2}\left(H^{\tau_{n-1} \tau_{n}} \circ m x^{n}\right) \quad$ such that

$$
\left\|_{\tau_{n-1} \leq t \leq \tau_{n}}\left|\left(x_{t}^{n}-x_{\tau_{n-1}^{n}-1}\right)-\Phi_{t}\left(f^{n}, g^{n}, h^{n}\right)\right|\right\|_{L_{1}^{2}<\epsilon / 2^{n}}
$$

Put $f^{\epsilon}=\sum_{n=1}^{\infty} \mathbf{I}_{\left[\tau_{n-1}, \tau_{n}\right)} f^{n}, g^{e}=\sum_{n=1}^{\infty} \mathbf{I}_{\left[\tau_{n-1}, \tau_{n}\right)} g^{n}$ and $h^{\epsilon}=\sum_{n=1}^{\infty} \mathbf{I}_{\left[\tau_{n-1}, \tau_{n}\right)} h^{n}$. By the decomposability (see [9], [10]) of $\varphi^{2}(F \circ m x), \varphi^{2}(G \circ m x)$ and $\varphi_{q}^{2}(h \circ m x)$, we get $f^{\epsilon} \in \varphi^{2}(F \circ m x), g^{\epsilon} \in \varphi^{2}(G \circ m x)$ and $h^{\epsilon} \in \varphi_{q}^{2}(H \circ m x)$. Moreover

$$
\begin{aligned}
& \left\|x-\Phi\left(f^{\epsilon}, g^{\epsilon}, h^{\epsilon}\right)\right\|_{\ell} \\
& \leq \| \sum_{n=1}^{\infty} \quad \sup _{\tau_{n-1} \leq t \leq \tau_{n}} \mid\left(x_{t}^{n}-x_{\tau_{n-1}^{n}-1}-\Phi_{t}\left(f^{n}, g^{n}, h^{n}\right) \mid \|_{L_{1}^{2}}\right. \\
& \leq \sum_{n=1}^{\infty} \| \quad \sup _{\tau_{n-1} \leq t \leq \tau_{n}} \mid\left(x_{t}^{n}-x_{\tau_{n-1}-1}^{n-1}-\Phi_{t}\left(f^{n}, g^{n}, h^{n}\right) \mid \|_{L_{1}^{2}}<\epsilon .\right.
\end{aligned}
$$

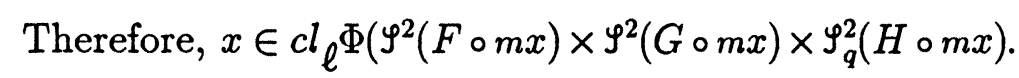

In what follows we shall deal with $F=\left\{\left(F_{t}(x)\right)_{t \geq 0}: x \in \mathbb{R}^{n}\right\}$, $G=\left\{\left(G_{t}(x)\right)_{t \geq 0}: \quad x \in \mathbb{R}^{n}\right\} \quad$ and $\left.\quad H=\left\{H_{t, z}(x)\right)_{t \geq 0, z \in \mathbb{R}^{n:}} x \in \mathbb{R}^{n}\right\} \quad$ satisfying conditions $\left(\mathcal{A}_{1}\right)$ and any one of the following conditions.

$\left(\mathcal{A}_{2}\right) F, G$ and $H$ are such that set-valued functions $D \ni x \rightarrow(F \circ m x)_{t}(\omega) \subset \mathbb{R}^{n}$, $D \ni x \rightarrow(G \circ m x)_{t}(\omega) \subset \mathbb{R}^{n}$ and $D \ni x \rightarrow(H \circ m x)_{t, z}(\omega) \subset \mathbb{R}^{n}$ are w.-w.s.u.s.c. on $D$, i.e., for every $x \in D$ and every sequence $\left(x_{n}\right)$ of $\left(D,\|\cdot\|_{\ell}\right)$ converging weakly to $x$, one has $\bar{h}\left(\iint_{A}\left(F \circ m x_{n}\right)_{t} d t d P\right.$, $\left.\iint_{A}(F \circ m x)_{t} d t d P\right) \rightarrow 0, \quad \bar{h}\left(\iint_{A}\left(G \circ m x_{n}\right)_{t} d t d P, \quad \iint_{A}(G \circ m x)_{t} d t d P\right) \rightarrow 0$ and $\bar{h}\left(\iiint_{B}\left(H \circ m x_{n}\right)_{t, z} d t q(d z) d P, \iiint_{B}(H \circ m x)_{t, z} d t q(d z) d P\right) \rightarrow 0$. 
$\left(\mathcal{A}_{3}\right) F, G$ and $H$ are such that set-valued functions $D \ni x \rightarrow(F \circ m x)_{t}(\omega) \subset \mathbb{R}^{n}$, $D \ni x \rightarrow(G \circ m x)_{t}(\omega) \subset \mathbb{R}^{n}$ and $D \ni x \rightarrow(H \circ m x)_{t, z}(\omega) \subset \mathbb{R}^{n}$ are s. - w.s.l.s.c. on $D$, i.e., for every $x \in D$ and every sequence $\left(x_{n}\right)$ of $\left(D,\|\cdot\|_{\ell}\right)$ converging weakly to $x$, one has $\bar{h}\left((F \circ m x)_{t}(\omega),\left(F \circ m x^{n}\right)_{t}(\omega)\right) \rightarrow 0$, $\bar{h}\left((G \circ m x)_{t}(\omega),\left(G \circ m x^{n}\right)_{t}(\omega)\right) \rightarrow 0$ and $\bar{h}\left((H \circ m x)_{t, z}(\omega), \quad\left(H \circ m x^{n}\right)_{t, z}(\omega)\right) \rightarrow 0$ a.e.

$\left(\mathcal{A}_{4}\right):$ There are $k, \ell \in \mathcal{L}_{1}^{2}$ and $m \in W_{1}^{2}$ such that $\left\|\int_{0}^{\infty} h\left[(F \circ m x)_{t},(F \circ m y)_{t}\right] d t\right\|_{L_{1}^{2}} \leq$ $E \int_{0}^{\infty} k_{t}\left|x_{t}-y_{t}\right| d t,\|h(G \circ m x, G \circ m y)\|_{\mathcal{L}_{1}^{2}} \leq E \int_{0}^{\infty} \ell_{t}\left|x_{t}-y_{t}\right| d t$ and $\|h(H \circ m x, H \circ m y)\|_{W_{1}^{2}} \leq E \int_{0}^{\infty} \int_{\mathbb{R}^{n}} m_{t, z}\left|x_{t}-y_{t}\right| d t q(d z)$ for $x, y \in D$.

$\left(\mathcal{A}_{4}^{\prime}\right)$ There are $k, \ell \in L^{2}\left(\mathscr{B}_{+}\right)$and $m \in L^{2}\left(\mathscr{B}_{+} \times \mathfrak{B}^{n}\right)$ such that $h\left(F_{t}\left(x_{2}\right)(\omega)\right.$, $\left.F_{t}\left(x_{1}\right)(\omega)\right) \leq k(t)\left|x_{1}-x_{2}\right|, \quad h\left(G_{t}\left(x_{2}\right)(\omega), G_{5}\left(x_{1}\right)(\omega)\right) \leq \ell(t)\left|x_{1}-x_{2}\right| \quad$ and $h\left(H_{t, z}\left(x_{2}\right)(\omega), \quad H_{t, z}\left(x_{1}\right)(\omega)\right) \leq m(t, z)\left|x_{1}-x_{2}\right| \quad$ a.e., each $t \geq 0 \quad$ and $x_{1}, x_{2} \in \mathbb{R}^{n}$.

It is clear that the upper (lower) semicontinuity of $F, G$ and $H$ does not imply their weak (strong) - weak sequential upper (lower) semicontinuity presented above. We shall show that in some special cases, i.e., for concave (convex, resp.), set-valued mappings such implication holds true. Recall a setvalued mapping $\mathfrak{R}$, defined on a locally convex topological space $\left(X, \mathscr{T}_{X}\right)$ with values in a normed space is said to be concave (convex) if $\mathscr{R}\left(\alpha x_{1}+\beta x_{2}\right) \subset$ $\alpha \Re\left(x_{1}\right)+\beta \Re_{0}\left(x_{2}\right)\left(\alpha \Re\left(x_{1}\right)+\beta \Re\left(x_{2}\right) \subset \Re_{(}\left(\alpha x_{1}+\beta x_{2}\right)\right)$, for every $x_{1}, x_{2} \in X$ and $\alpha, \beta \in[0,1]$ satisfying $\alpha+\beta=1$.

Lemma 1: $\quad$ Suppose $F, G$ and $H$ satisfy $\left(\mathcal{A}_{1}\right)$ with $p=1$, take on convex values and are concave (convex) with respect to $x \in \mathbb{R}^{n}$. If moreover $F, G$ and $H$ are u.s.c. (l.s.c.) with respect to $x \in \mathbb{R}^{n}$ then they are w.-w.s.u.s.c. (s.w.s.l.s.c.).

Proof: Let $x \in D$ be fixed and let $\left(x^{n}\right)$ be a sequence of $D$ weakly converging to $x$. Denote $K_{p}(t, \omega, y):=-s\left(p, F_{t}\left(y_{t}\right)(\omega)\right)$ for $p \in \mathbb{R}^{n}, y \in D, t \geq 0$ and $\omega \in \Omega$. We shall show that for every $A \in \mathcal{P}$ and every $p \in \mathbb{R}^{n}$ one has

$$
\iint_{A} K_{p}(t, \omega, x) d t d P \leq \operatorname{limin}_{n \rightarrow \infty} \iiint_{A} K_{p}\left(t, \omega, x^{n}\right) d t d P,
$$

which is equivalent to the weak-weak sequential upper semicontinuity of $F$ at $x \in D$ in the sense defined in $\left(\mathcal{A}_{2}\right)$. Similarly, the weak-weak sequential upper 
semicontinuity of $G$ and $H$ can be verified.

Let $A \in \mathscr{P}, \quad p \in \mathbb{R}^{n}$ be given. Denote $j_{n}=\iint_{A} K_{p}\left(t, \omega, x^{n}\right) d t d P$ for $n=1,2, \ldots$ and put $i:=\liminf _{n \rightarrow \infty} \iint_{A} K_{p}\left(t, \omega, x^{n}\right) d t d P$. By taking a suitable subsequence, say $\left(n_{k}\right)$ of $(n)$ we may well assume that $j_{n_{k}} \rightarrow i$ as $k \rightarrow \infty$. By the Banach and Mazur theorem (see [2]) for every $s=1,2, \ldots$ there are numbers $\alpha_{k}^{s} \geq 0$ with $k=1,2, \ldots, N$ and $N=1,2, \ldots$ satisfying $\sum_{k=1}^{N} \alpha_{k}^{s}=1$ and such that $\left\|z_{N}^{s}-x\right\|_{\ell} \rightarrow 0$ as $N \rightarrow 0$, where $z_{N}^{s}(t, \omega)=\sum_{k=1}^{N} \alpha_{k}^{s} x_{t}^{n_{k}+s}(\omega)$. By the definition of the norm $\|\cdot\|_{\ell}$ there is a subsequence, say again $\left(z_{N}^{s}\right)$, of $\left(z_{N}\right)$ such that

$$
\begin{array}{r}
\sup _{t \geq 0}\left|z_{N}^{s}(t, \omega)-x_{t}(\omega)\right| \rightarrow 0 \text { a.s. for } s=1,2, \ldots \text { Put } \\
\eta_{N}^{s}:=\sum_{k=1}^{N} \alpha_{k}^{s} K_{p}\left(\cdot, \cdot, x^{n_{k+s}}\right), \\
j_{k}^{s}=\iint_{A} K_{p}\left(t, \omega, x^{n_{k}+s}\right) d t d P
\end{array}
$$

and let $\delta_{s}=\max _{N \geq s+1} \max _{1 \leq k \leq N}\left|j_{k}^{s}-i\right|$ for $s=1,2, \ldots$. We have $\delta_{s} \rightarrow 0$ as $s \rightarrow \infty$. By the uniform square boundedness of $F$ there is $m_{F} \in \mathcal{L}_{1}^{2}$ such that $\eta_{N}^{s} \geq-m_{F}$ a.e. for $N, s=1,2, \ldots$. Therefore, $\liminf f_{N \rightarrow \infty} \eta_{N}^{s} \geq-m_{F}$ a.e. for $s=1,2, \ldots$. Then by Fatou's lemma one obtains

$$
\iint_{A} \operatorname{limin}_{N \rightarrow \infty} \eta_{N}^{s} d t d P \leq \liminf _{N \rightarrow \infty} \iint_{A} \eta_{N}^{s} d t d P \leq i+\delta_{s}
$$

for $s=1,2, \ldots$, because for every $s=1,2, \ldots$, we have $i-\delta_{s} \leq \iint_{A} \eta_{N}^{s} d t d P \leq i+\delta_{s}$. Taking $\quad \eta=\liminf f_{s \rightarrow \infty}\left[\liminf f_{N \rightarrow \infty} \eta_{N}^{s}\right]$ a.e., we get $\eta \geq-m_{F} \quad$ a.e. and $\iint_{A} \eta d t d P \leq i$. We shall verify that we also have $K(t, \omega, x) \leq \eta(t, \omega)$ for a.e. $(t, \omega) \in \mathbb{R}_{+} \times \Omega$. Indeed, by upper semicontinuity of $F$ with respect to $x \in \mathbb{R}^{n}$, a real valued function $x \rightarrow-s\left(p, F_{t}(x)\right)$ is lower semicontinuous on $\mathbb{R}^{n}$, a.s. for every $t \geq 0$ and $p \in \mathbb{R}^{n}$. Therefore for every $m, s=1,2, \ldots$ there is $M \geq 1$ such that

$$
-s\left(p, F_{t}\left(x_{t}\right)\right)-\frac{1}{m}<-s\left(p, F_{t}\left(\sum_{k=1}^{N} \alpha_{k}^{s} x_{t}^{n} k+s\right)\right)
$$

a.s. for every $t \geq 0$ and $N \geq M$. Hence, by the properties of $F$, it follows

$$
-s\left(p, F_{t}\left(x_{t}\right)\right)-\frac{1}{m}<\sum_{k=1}^{N} \alpha_{k}^{s}\left[-s\left(p, F_{t}\left(x_{t}^{n_{k}+s}\right)\right)\right]=: \eta_{N}^{s}(t, \cdot)
$$

a.s. for $t \geq 0, s, m=1,2 \ldots$ and $N \geq M$. Therefore, for $m=1,2, \ldots$ almost everywhere, one gets 


$$
K_{p}(\cdot, \cdot, x)-\frac{1}{m} \leq \operatorname{limin}_{s \rightarrow \infty} f\left[\operatorname{limin}_{N \rightarrow \infty} f \eta_{N}^{s}\right]=\eta
$$

Finally, we get

$$
\iint_{A} K_{p}(t, \omega, x) d t d P \leq \iint_{A} \eta(t, \omega) d t d P \leq i
$$

\section{PROPERTIES OF SOLUTION SET}

We shall prove here the existence theorems for $S I(F, G, H)$. We show first that conditions $\left(\mathcal{A}_{1}\right)$ and anyone of conditions $\left(\mathcal{A}_{2}\right)-\left(\mathcal{A}_{4}\right)$ or $\left(\mathcal{A}_{4}^{\prime}\right)$ imply the existence of fixed points for the set-valued mappings $J_{6}$ and $J_{6}^{\alpha, \beta}$ defined above. Hence, by Propositions 4 and 5 , the existence theorems for $S I(F, G, H)$ will follow. We begin with the following lemmas.

Lemma 2: $\quad$ Assume $F, G$ and $H$ take on convex values, satisfy $\left(\mathcal{A}_{1}\right)$ with $p=2$ and $\left(\mathcal{A}_{2}\right)$. Then a set-valued mapping $\mathfrak{J}_{6}$ is u.s.c. as a multifunction defined on a locally convex topological Hausdorff space $\left(D, \sigma\left(D, D^{*}\right)\right)$ with nonempty values in $\left(D, \sigma\left(D, D^{*}\right)\right)$.

Proof: Let $C$ be a nonempty weakly closed subset of $D$ and select a sequence $\left(x^{n}\right)$ of $J_{6}-(C)$ weakly converging to $x \in D$. There is a sequence $\left(y^{n}\right)$ of $C$ such that $y^{n} \in \mathcal{H}\left(x^{n}\right)$ for $n=1,2, \ldots$. By the uniform square-integrable boundedness of $F, G$ and $H$, there is a convex weakly compact subset $\mathfrak{B} \subset \mathcal{L}_{n}^{2} \times \mathcal{L}_{n}^{2} \times \mathscr{W}_{n}^{2}$ such that $\mathfrak{J}\left(x^{n}\right) \subset \Phi(\mathfrak{B})$. Therefore, $y^{n} \in \Phi(\mathfrak{B})$, for $n=1,2, \ldots$ which, by the weak compactness of $\Phi(\mathfrak{B})$, implies the existence of a subsequence, say for simplicity $\left(y^{k}\right)$, of $\left(y^{n}\right)$ weakly converging to $y \in \Phi(\mathfrak{B})$. We have $y^{k} \in \mathcal{J} \mathcal{G}\left(x^{k}\right)$ for $k=1,2, \ldots$ Let $\left(f^{k}, g^{k}, h^{k}\right) \in \mathcal{Y}^{2}\left(F \circ m x^{k}\right) \times \varphi^{2}\left(G \circ m x^{k}\right) \times \varphi_{q}^{2}\left(H \circ m x^{k}\right)$ be such that $\Phi\left(f^{k}, g^{k}, h^{k}\right)=y^{k}$, for each $k=1,2, \ldots$. We have of course $\left(f^{k}, g^{k}, h^{k}\right) \in \mathfrak{B}$. Therefore, there is a subsequence, say again $\left\{\left(f^{k}, g^{k}, h^{k}\right)\right\}$ of $\left\{\left(f^{k}, g^{k}, h^{k}\right)\right\}$ weakly converging in $\mathcal{L}_{n}^{2} \times \mathcal{L}_{n}^{2} \times \mathcal{W}_{n}^{2}$ to $(f, g, h) \in \mathfrak{B}$. Now, for every $A \in \mathcal{P}$ one obtains

$$
\begin{gathered}
\operatorname{dist}\left(\iint_{A} f_{t} d t d P, \iint_{A} F_{t}(x) d t d P\right) \leq \\
\leq\left|\iint_{A}\left[f_{t}-f_{t}^{k}\right] d t d P\right|+\operatorname{dist}\left(\iint_{A} f_{t}^{k} d t d P, \iint_{A} F_{t}\left(x_{k}\right) d t d P\right)
\end{gathered}
$$




$$
+\bar{h}\left(\iint_{A} F_{t}\left(x^{k}\right) d t d P, \iint_{A} F_{t}(x) d t d P\right) .
$$

Therefore (see [8], Lemma 4.4) $f \in \varphi^{2}(F \circ m x)$. Quite similarly, we also get $t \in \mathscr{\varphi}^{2}(G \circ m x)$ and $h \in \Psi_{q}^{2}(H \circ m x)$. Thus, $\Phi(f, g, h) \in J 6(x)$, which implies $y \in \mathfrak{J}(x)$. On the other hand we also have $y \in C$, because $C$ is weakly closed. Therefore, $x \in J_{6}-(C)$. Now the result follows immediately from Eberlein and Šmulian's theorem.

Lemma 3: $\quad$ Assume $F, G$ and $H$ take on convex values, satisfy $\left(\mathcal{A}_{1}\right)$ with $p=2$ and $\left(\mathcal{A}_{3}\right)$. Then a set-valued mapping $\mathfrak{H}_{6}$ is l.s.c. as a multifunction defined on a locally convex topological Hausdorff space $\left(D, \sigma\left(D, D^{*}\right)\right)$ with nonempty values in $\left(D, \sigma\left(D, D^{*}\right)\right)$.

Proof: Let $C$ be a nonempty weakly closed subset of $D$ and $\left(x^{n}\right)$ a sequence of $\mathfrak{H}_{-}(C)$ weakly converging to $x \in D$. Select arbitrarily $y \in \mathcal{H}_{G}(x)$ and suppose $(f, g, h) \in \varphi^{2}(F \circ m x) \times \varphi^{2}(G \circ m x) \times \varphi_{q}^{2}(H \circ m x)$ is such that $y=\Phi(f, g, h)$. Let $\left(f^{n}, g^{n}, h^{n}\right) \in \varphi^{2}\left(F \circ m x^{n}\right) \times \varphi^{2}\left(G \circ m x^{n}\right) \times \varphi_{q}^{2}\left(H \circ m x^{n}\right)$ be such that

$$
\begin{aligned}
\left|f_{t}(\omega)-f_{t}^{n}(\omega)\right| & =\operatorname{dist}\left(f_{t}(\omega),\left(F \circ m x^{n}\right)_{t}(\omega)\right), \\
\left|g_{t}(\omega)-g_{t}^{n}(\omega)\right| & =\operatorname{dist}\left(g_{t}(\omega),\left(G \circ m x^{n}\right)_{t}(\omega)\right)
\end{aligned}
$$

and $\left|h_{t, z}(\omega)-g_{t, z}^{n}(\omega)\right|=\operatorname{dist}\left(h_{t, z}(\omega),\left(H \circ m x^{n}\right)_{t, z}(\omega)\right)$ on $\mathbb{R}_{+} \times \Omega$ and $\mathbb{R}_{+} \times \Omega \times \mathbb{R}^{n}$, respectively, for each $n=1,2, \ldots$. By virtue of $\left(\mathcal{A}_{3}\right)$ one gets $\left|f_{t}(\omega)-f_{t}^{n}(\omega)\right| \rightarrow 0$, $\left|g_{t}(\omega)-g_{t}^{n}(\omega)\right| \rightarrow 0$ and $\left|h_{t, z}(\omega)-h_{t, z}^{n}(\omega)\right| \rightarrow 0$ a.e., as $n \rightarrow \infty$. Hence, by $\left(\mathcal{A}_{1}\right)$ we can easily see that a sequence $\left(y_{n}\right)$, defined by $y^{n}=\Phi\left(f^{n}, g^{n}, h^{n}\right)$, weakly converges to $y$. But $y^{n} \in J_{G}\left(x^{n}\right) \subset C$ for $n=1,2, \ldots$ and $C$ is weakly closed. Then $y \in C$ which implies $\mathfrak{J}_{6}(x) \subset C$. Thus $x \in \mathfrak{J}_{-}(C)$.

Lemma 4. Suppose $F, G$ and $H$ satisfy $\left(\mathcal{A}_{1}\right)$ and $\left(\mathcal{A}_{4}\right)$ or $\left(\mathcal{A}_{4}^{\prime}\right)$. Then $H\left(\mathfrak{J}_{6}(x), \mathfrak{J}_{G}(y)\right) \leq L\|x-y\|_{\ell}$ or $H\left(\mathfrak{J}_{\mathcal{G}}(x), \mathfrak{J}_{\mathcal{G}}(y)\right) \leq L^{\prime}\|x-y\|_{\ell}$, respectively, for every $x, y \in D$, where $H$ is the Hausdorff metric induced by the norm $\|\cdot\|_{\ell}$ ' $L=\left\|\int_{0}^{\infty} k_{t} d t\right\|_{L_{1}^{2}}+2\left\|\int_{0}^{\infty} \ell_{t} d t\right\|_{L_{1}^{2}}+2\left\|\int_{0}^{\infty} \int_{\mathbb{R}^{n}} m_{\tau, z} d \tau q(d z)\right\|_{L_{1}^{2}} \quad$ and $\quad L^{\prime}=|k|_{1}$ $+2|\ell|_{2}+2\|m\|_{2}$.

Proof: Let $x, y \in D$ be given and let $u \in \mathcal{J} G(x)$. For every $\epsilon>0$, there is $\left(f^{\epsilon}, g^{\epsilon}, h^{\epsilon}\right) \in \varphi^{2}(F \circ m x) \times \varphi^{2}(G \circ m x) \times \varphi_{q}^{2}(H \circ m x)$ such that $\left\|u-\Phi\left(f^{\epsilon}, g^{\epsilon}, h^{\epsilon}\right)\right\|_{\ell}$ $<\epsilon$. Select now $\left(\tilde{f}^{\epsilon}, \tilde{g}^{\epsilon}, \tilde{h}^{\epsilon}\right) \in \varphi^{2}(F \circ m y) \times \varphi^{2}(G \circ m y) \times \varphi_{q}^{2}(H \circ m y)$ such that 


$$
\begin{aligned}
\left|f_{t}^{\epsilon}(\omega)-\tilde{f}_{t}^{\epsilon}(\omega)\right| & =\operatorname{dist}\left(f_{t}^{\epsilon}(\omega),(F \circ m y)_{t}(\omega)\right) \\
\left|g_{t}^{\epsilon}(\omega)-\tilde{g}_{t}^{\epsilon}(\omega)\right| & =\operatorname{dist}\left(g_{t}^{\epsilon}(\omega),(G \circ m y)_{t}(\omega)\right)
\end{aligned}
$$

and

$\left|h_{t, z}^{\epsilon}(\omega)-\tilde{h}_{t, z}^{\epsilon}(\omega)\right|=\operatorname{dist}\left(h_{t, z}^{\epsilon}(\omega),(H \circ m y)_{t, z}(\omega)\right)$ on $\mathbb{R}_{+} \times \Omega$ and $\mathbb{R}_{+} \times \Omega \times \mathbb{R}^{n}$, respectively. Now, by $\left(\mathcal{A}_{4}\right)$ it follows

$$
\begin{aligned}
& E\left[\sup _{t \geq 0}\left|\int_{0}^{t}\left(f_{\tau}^{\epsilon}-\tilde{f}_{\tau}^{\epsilon}\right) d \tau\right|\right]^{2} \leq E\left[\int_{0}^{\infty}\left|f_{\tau}^{\epsilon}-\tilde{f}_{\tau}^{\epsilon}\right| d \tau\right]^{2} \\
\leq & E\left[\int_{0}^{t} \bar{h}\left((F \circ m x)_{\tau},(F \circ m y)_{\tau}\right) d \tau\right]^{2} \leq\left(E \int_{0}^{t} k_{\tau}\left|x_{\tau}-y_{\tau}\right| d \tau\right)^{2} \\
\leq & {\left[E\left(\sup _{t \geq 0}\left|x_{t}-y_{t}\right| \cdot \int_{0}^{\infty} k_{\tau} d \tau\right)\right]^{2} \leq E\left(\int_{0}^{\infty} k_{\tau} d \tau\right)^{2} \cdot\|x-y\|_{\ell}^{2} . }
\end{aligned}
$$

Similarly, by Doob's inequality, we obtain

$$
\begin{aligned}
& E\left[\sup _{t \geq 0}\left|\int_{0}^{t}\left(g_{\tau}^{\epsilon}-\tilde{g}_{\tau}^{\epsilon}\right) d w_{\tau}\right|\right]^{2} \leq 4 E \int_{0}^{\infty}\left|g_{\tau}^{\epsilon}-\tilde{g}_{\tau}^{\epsilon}\right|^{2} d \tau \\
& \leq 4 E \int_{0}^{\infty}\left[\bar{h}\left((G \circ m x)_{\tau},(G \circ m y)_{\tau}\right)\right]^{2} d \tau \leq 4\left(E \int_{0}^{\infty} \ell_{\tau}\left|x_{\tau}-y_{\tau}\right| d \tau\right)^{2} \\
& \leq 4\left[E\left(\sup _{t \geq 0}\left|x_{t}-y_{t}\right| \cdot \int_{0}^{\infty} \ell_{\tau} d \tau\right)\right]^{2} \leq 4 E\left(\int_{0}^{\infty} \ell_{\tau} d \tau\right)^{2}\|x-y\|^{2} .
\end{aligned}
$$

Quite similarly, we also get

$$
\begin{aligned}
& \left.E\left[\sup _{t \geq 0} \mid \int_{0}^{t} \int_{\mathbb{R}^{n}} h_{\tau}^{\epsilon}-\tilde{h}_{\tau, z}^{\epsilon}\right) \tilde{\nu}(d \tau, d z)\right]^{2} \\
& \leq 4 E\left(\int_{0}^{\infty} \int_{\mathbb{R}^{n}} m_{\tau, z} d \tau q(d z)\right)^{2} \cdot\left\|x_{\tau}-y_{\tau}\right\|_{\ell}^{2} .
\end{aligned}
$$

Therefore

$$
\| u-\Phi\left(\tilde{f}^{\epsilon}, \tilde{g}^{\epsilon}, \tilde{h}^{\epsilon} \|_{\ell}\right.
$$




$$
\leq\left\|u-\Phi\left(f^{\epsilon}, g^{\epsilon}, h^{\epsilon}\right)\right\|_{\ell}+\left\|\Phi\left(f^{\epsilon}, g^{\epsilon}, h^{\epsilon}\right)-\Phi\left(\tilde{f}^{\epsilon}, \tilde{g}^{\epsilon}, \tilde{h}^{\epsilon}\right)\right\|_{\ell} \leq \epsilon+L\|x-y\|_{\ell}
$$

where $L$ is such as above. This implies $\bar{H}(\mathfrak{J G}(x), \mathfrak{J K}(y)) \leq L\|x-y\|_{\ell}$. Quite

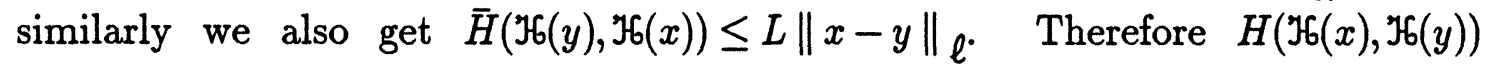
$\leq L|x-y|_{\ell}$. Using conditions $\left(\mathcal{A}_{4}^{\prime}\right)$ instead of $\left(\mathcal{A}_{4}\right)$ we also get $H\left(\mathfrak{J}_{6}(y), \mathfrak{T} \mathfrak{K}(x)\right)$ $\leq L^{\prime}\|x-y\|_{\ell}$.

Lemma 5: $\quad$ Suppose $F, G$ and $H$ satisfy $\left(\mathcal{A}_{1}\right)$ and $\left(\mathcal{A}_{4}\right)$ or $\left(\mathcal{A}_{4}^{\prime}\right)$. Then for every $0 \leq \alpha<\beta<\infty$ one has $H\left(\mathcal{J G}_{6}^{\alpha, \beta}(x), \mathcal{J G}_{6}^{\alpha, \beta}(y)\right) \leq L_{\alpha \beta}\|x-y\|_{\ell}$ or $H\left(\mathfrak{J}^{\alpha, \beta}(x), J_{6}^{\alpha, \beta}(y)\right) \leq L_{\alpha \beta}^{\prime}|x-y|_{\ell}$, respectively, for every $x, y \in D^{\alpha, \beta}$, where $H$ is a Hausdorff metric induced by the norm $\|\cdot\|_{\ell^{\prime}} L_{\alpha, \beta}=\left\|\int_{0}^{\infty} \bigsqcup_{[\alpha, \beta]}(t) k_{t} d t\right\|_{L_{1}^{2}}$ $+2\left\|\int_{0}^{\infty} \mathbf{l}_{[\alpha, \beta]}(t) \ell_{t} d t\right\|_{L_{1}^{2}}+2\left\|\int_{0}^{\infty} \int_{\mathbb{R}^{n}} \mathbf{l}_{[\alpha, \beta]}(t) m_{t, z} d t q(d z)\right\|_{L_{1}^{2}}$ and $L_{\alpha, \beta}^{\prime}=\left|\mathbf{u}_{[\alpha, \beta]} k\right|_{1}$ $+2\left|\mathbf{u}_{[\alpha, \beta]} \ell\right|_{2}+2\left\|\mathbf{u}_{[\alpha, \beta]} m\right\|_{2}$.

Proof: The proof follows immediately from Lemma 4 applied to $F^{\alpha \beta}=\mathbf{!}_{[\alpha, \beta]} F, G^{\alpha \beta}=\mathbf{!}_{[\alpha, \beta[} G$ and $H^{\alpha \beta}=\mathbf{!}_{[\alpha, \beta]} H$.

Immediately from Lemma 2 and the Kakutani and Fan fixed point theorem the following result follows.

Lemma 6: If $F, G$ and $H$ take on convex values and satisfy $\left(\mathcal{A}_{1}\right)$ and $\left(\mathcal{A}_{2}\right)$, then $S(F, G, H) \neq \emptyset$.

Proof: Let $\quad \mathfrak{B}=\left\{(f, g, h) \in \mathcal{L}_{n}^{2} \times \ell_{n}^{2} \times W_{n}^{2}:\left|f_{t}(\omega)\right| \leq\left\|F_{t}(\omega)\right\|\right.$, $\left|g_{t}(\omega)\right| \leq\left\|G_{t}(\omega)\right\|,\left|h_{t, z}(\omega)\right| \leq\left\|H_{t, z}(\omega)\right\|$ and put $\mathscr{K}=\Phi(\mathfrak{B})$. It is clear that $\mathscr{S}$ is a nonempty convex weakly compact subset of $D$ such that $\mathcal{H}(x) \subset \mathscr{K}$ for $x \in D$. By (ii) of Proposition $1, J(x)$ is a convex and weakly compact subset of $D$, for each $x \in D$. By Lemma $2, J_{6}$ is u.s.c. on a locally convex topological Hausdorff space $\left(D, \sigma\left(D, D^{*}\right)\right)$. Therefore, by the Kakutani and Fan fixed point theorem, we get $S(F, G, H) \neq \emptyset$.

Lemma 7. If $F, G$ and $H$ take on convex values and satisfy $\left(\mathcal{A}_{1}\right)$ and $\left(\mathcal{A}_{3}\right)$, then $S(F, G, H) \neq \emptyset$.

Proof. Let $\mathfrak{T} 6$ be as in Lemma 6. By virtue of Lemma 3, $\mathfrak{J}_{6}$ is l.s.c. as a set-valued mapping from a paracompact space 96 considered with its relative topology induced by a weak topology $\sigma\left(D, D^{*}\right)$ on $D$ into a Banach space $\left(D,\|\cdot\|_{\ell}\right)$. By $(i i)$ of Proposition $1, \mathfrak{J}(x)$ is a closed and convex subset of $D$, for 
each $x \in \mathscr{T 6}$. Therefore, by Michael's theorem, there is a continuous selection $f: \mathscr{G} \rightarrow D$ for $J_{6}$. But $J_{6}(\mathscr{W}) \subset \mathscr{G}$. Then $f$ maps $\mathscr{T}$ into itself and is continuous with respect to the relative topology on 96 , defined above. Therefore, by the Schauder and Tikhonov fixed point theorem, there is $x \in \mathscr{K}$ such that $x=f(x) \in J_{G}(x)$.

Lemma 8. If $F, G$ and $H$ satisfy $\left(\mathcal{A}_{1}\right)$ and $\left(\mathcal{A}_{4}\right)$ or $\left(\mathcal{A}_{4}^{\prime}\right)$ then $S(F, G, H) \neq \emptyset$.

Proof. Let $\left(\tau_{n}\right)_{n=1}^{\infty}$ be a sequence of positive numbers increasing to $+\infty$. Select a positive number $\sigma$ such that $L_{k \sigma,(k+1) \sigma}<1$ or $L_{k \sigma,(k+1) \sigma}^{\prime}<1$, respectively, for $k=0,1, \ldots$, where $L_{k \sigma,(k+1) \sigma}$ and $L_{k \sigma,(k+1) \sigma}^{\prime}$ are as in Lemma 5 . Suppose a positive integer $n_{1}$ is such that $n_{1} \sigma<\tau_{1} \leq\left(n_{1}+1\right) \sigma$. By virtue of Lemma $5, J_{6}^{k \sigma,(k+1) \sigma}$ is a set-valued contraction for every $k=0,1, \ldots$. Therefore, by the Covitz and Nadler fixed point theorem, there is $z^{1} \in S^{0, \sigma}(F, G, H)$. By the same argument, there is $z^{2} \in z_{\sigma}^{1}+S^{\sigma, 2 \sigma}(F, G, H)$, because $z_{\sigma}^{1}+J_{6}^{\sigma, 2 \sigma}$ is again a set-valued contraction mapping. Continuing the above procedure we can finally find a $z^{n_{1}+1} \in z_{n_{1} \sigma}^{n_{1}}+S^{n_{1} \sigma, \tau_{1}}(F, G, H)$. Put

$$
\begin{gathered}
x^{1}=\sum_{k=0}^{n_{1}-1} \mathbb{1}_{[k \sigma,(k+1) \sigma)}\left(z^{k+1}-z_{k \sigma}^{k}\right) \\
+\mathbb{I}_{\left[n_{1} \sigma, \tau_{1}\right]}\left(z^{n_{1}+1}-z_{n_{1} \sigma}^{n_{1}}\right)+\mathbb{I}_{\left(\tau_{1}, \infty\right)}\left(z_{\tau_{1}}^{n_{1}+1}-z_{n_{1} \sigma}^{n_{1}}\right),
\end{gathered}
$$

where $z_{0}^{0}=0$. Similarly, as in the proof of Proposition 6 , we can easily verify that $x^{1} \in S^{0, \tau_{1}}(F, G, H)$. Repeating the above procedure to the interval $\left[\tau_{1}, \tau_{2}\right]$, we can find $x^{2} \in x_{\tau_{1}}^{1}+S^{\tau_{1}, \tau_{2}}(F, G, H)$. Continuing this process we can define a sequence $\left(x^{n}\right)$ of $D$ satisfying the conditions of Proposition 6 . Therefore $S(f, G, H) \neq \emptyset$.

Now as a corollary of Proposition 4 and Lemmas 6-8, the following results follow.

Theorem 1. Suppose $F, G$ and $H$ take on convex values, satisfy $\left(\mathcal{A}_{1}\right)$ and $\left(\mathcal{A}_{2}\right)$ or $\left(\mathcal{A}_{3}\right)$. Then $\Lambda_{0}(F, G, H) \neq \emptyset$.

Theorem 2. Suppose $F, G$ and $H$ satisfy $\left(\mathcal{A}_{1}\right)$ and $\left(\mathcal{A}_{4}\right)$ or $\left(\mathcal{A}_{4}^{\prime}\right)$ and 
take on convex values. Then $\Lambda_{0}(F, G, H) \neq \emptyset$.

From the stochastic optimal control theory point of view (see [6]), it is important to know whether the set $\Lambda_{0}(F, G, H)$ is at least weakly compact in $\left(D,\|\cdot\|_{\ell}\right)$. We have the following result dealing with this topic.

Theorem 3. Suppose $F, G$ and $H$ take on convex values and satisfy $\left(\mathcal{A}_{1}\right)$ and $\left(\mathcal{A}_{2}\right)$. Then $\Lambda_{0}(F, G, H)$ is a nonempty weakly compact subset of $\left(D,\|\cdot\|_{\ell}\right)$.

Proof. Nonemptiness of $\Lambda_{0}(F, G, H)$ follows immediately from Theorem 1. By virtue of Proposition 4 and the Eberein and Šmulian theorem for the weak compactness of $\Lambda_{0}(F, G, H)$, it suffices only to verify that $S(F, G, H)$ is sequentially weakly compact. But $S(F, G, H) \subset \Phi(\mathfrak{B})$, where $\mathfrak{B}$ is a weakly compact subset of $\ell_{n}^{2} \times \mathcal{L}_{n}^{2} \times W_{n}^{2}$ defined in Lemma 6 . Hence, by the properties of the linear mapping $\Phi$, the relative sequential weak compactness of $S(F, G, H)$ follows. Suppose $\left(x^{n}\right)$ is a sequence of $S(F, G, H)$ weakly converging to $x \in \Phi(\mathfrak{B})$, and let $\left(f^{n}, g^{n}, h^{n}\right) \in \varphi^{2}\left(F \circ m x^{n}\right) \times \mathcal{Y}^{2}\left(G \circ m x^{n}\right) \times \Psi_{q}^{2}\left(H \circ m x^{n}\right)$ be such that $x^{n}=\Phi\left(f^{n}, g^{n}, h^{n}\right)$, for $n=1,2, \ldots$ By the weak compactness of $\mathscr{B}$, there is a subsequence, denoted again by $\left\{\left(f^{n}, g^{n}, h^{n}\right)\right\}$, of $\left\{\left(f^{n}, g^{n}, h^{n}\right)\right\}$ weakly converging to $(f, g, h) \in \mathfrak{B}$. Similarly, as in the proof of Lemma 2, we can verify that $(f, g, h) \in \varphi^{2}(F \circ m x) \times \varphi^{2}(G \circ m x) \times \varphi_{q}^{2}(H \circ m x)$. This and the weak convergence of $\left\{\Phi\left(f^{n}, g^{n}, h^{n}\right)\right\}$ to $\Phi(f, g, h)$ imply that $x=\Phi(f, g, h) \in \mathcal{H}(x)$. Thus $x \in S(F, G, H) \square$

\section{REFERENCES}

[1] Ahmed, N.U., Optimal control of stochastic dynamical systems, Information and Control 22 (1973), 13-30.

[2] Alexiewicz, A., Functional Analysis, Monografie Matematyczne 49, Polish Scientif Publishers, Warszawa, Poland 1969.

[3] Chung, K.L., Williams, R.J., Introduction to Stochastic Integrals, Birkhäuser, BostonBasel 1983.

[4] Fleming, W.H., Stochastic control for small noise intensities, SIAM J. Contr. 9 (1971), 473-517.

[5] Fleming, W.H., Nisio, M., On the existence of optimal stochastic controls, J. Math. and Mech. 15 (1966), 777-794.

[6] Gihman, I.I., Skorohod, A.V., Controlled Stochastic Processes, Springer-Verlag, Berlin New York 1979. 
[7] Hiai, F., Umegaki, $\dot{H}$., Integrals, conditional expectations and martingales of multifunctions, J. Multivariate Anal. 7 (1977), 149-182.

[8] Ikeda, N., Watanabe, S., Stochastic Differential Equations and Diffusion Processes, North Holland Publ. Comp., Amsterdam-Tokyo 1981.

[9] Kisieliwicz, M., Differential Inclusions and Optimal Control, Kluwer Acad. Publ. and Polish Sci. Publ., Warszawa-Dordrecht-Boston-London 1991.

[10] Kisielewicz, M., Set-valued stochastic integrals and stochastic inclusions. Ann. Probab., (submitted for publication).

[11] Protter, Ph., Stochastic Integration and Differential Equations, Springer-Verlag, BerlinHeidelberg-New York 1990.

[12] Wentzel, A.D., Course of Theory of Stochastic Processes, Polish Scientific Publishers 1980 (in Polish). 


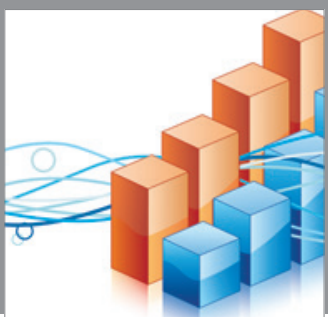

Advances in

Operations Research

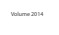

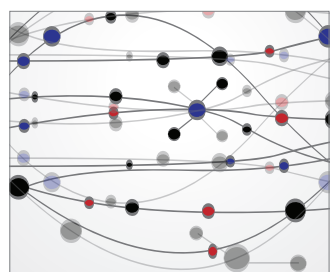

\section{The Scientific} World Journal
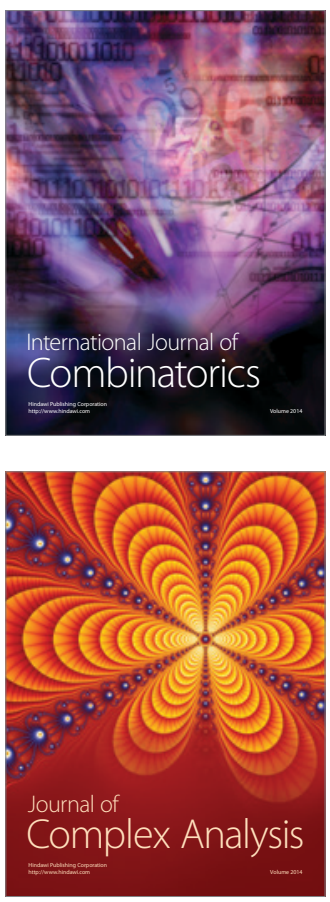

International Journal of

Mathematics and

Mathematical

Sciences
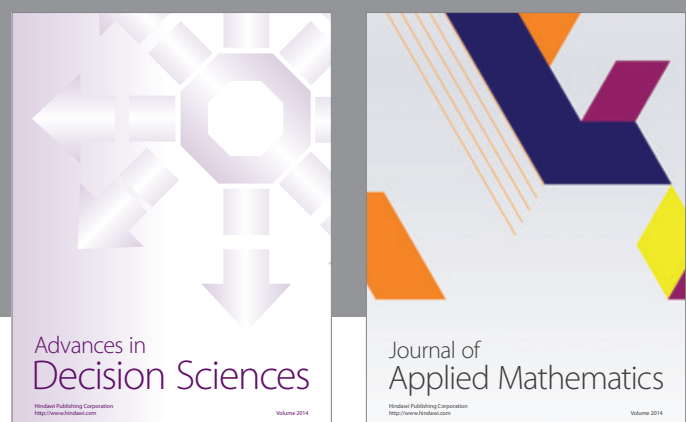

Journal of

Applied Mathematics
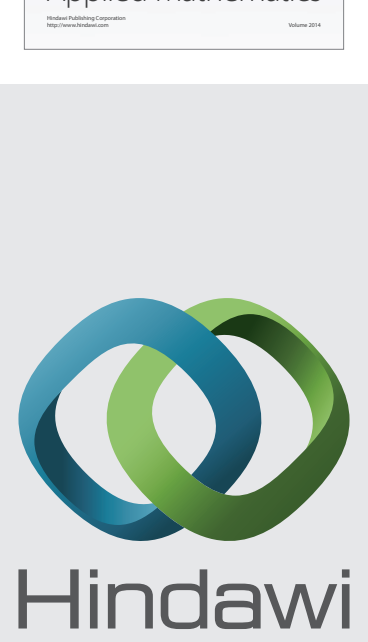

Submit your manuscripts at http://www.hindawi.com
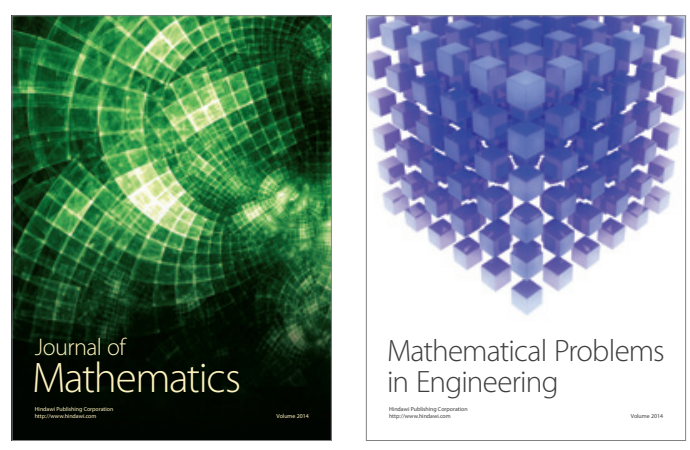

Mathematical Problems in Engineering
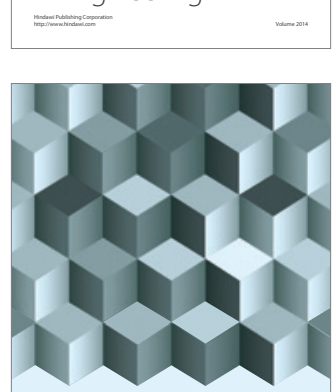

Journal of

Function Spaces
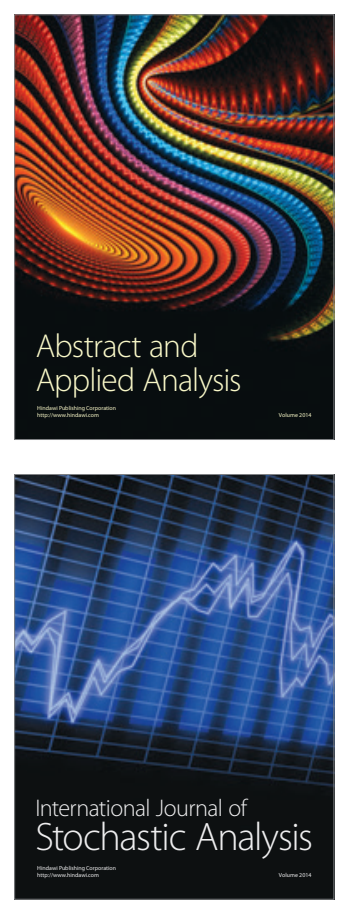

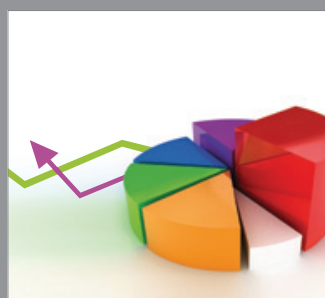

ournal of

Probability and Statistics

Promensencen
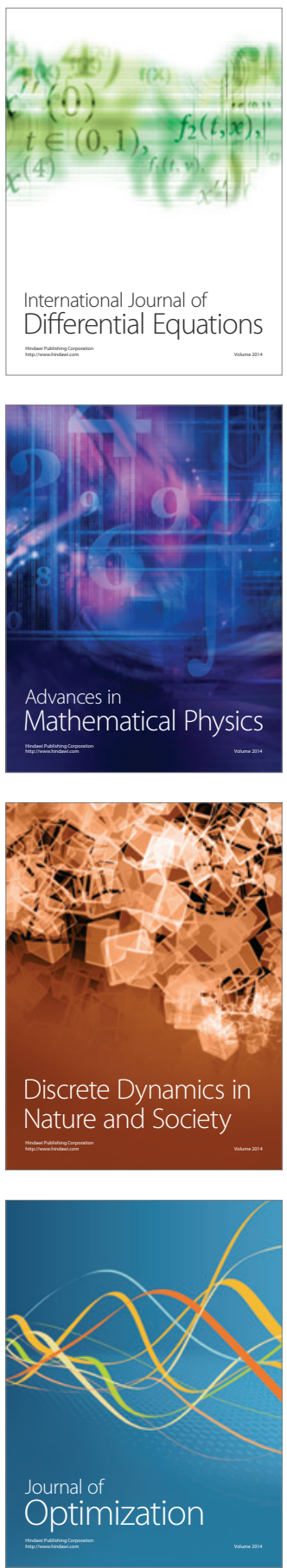